\title{
Peri-Operative Cerebral Regional Oxygen Saturation Variability and Short-Term Outcomes Following Cardiac Surgery in Neonates
}

\author{
University of Virginia

\section{Santina A. Zanelli} \\ University of Virginia \\ Michael C. Spaeder \\ University of Virginia
}

Maryam 0. Abubakar ( $\sim$ moa3yr@virginia.edu )

\section{Research Article}

Keywords: Near infrared spectroscopy (NIRS), congenital heart disease (CHD), cerebral region oxygenation saturation (crSO2), Peri-Operative, Short-Term Outcome, Cardiac Surgery, Neonates

Posted Date: August 31st, 2021

DOI: https://doi.org/10.21203/rs.3.rs-831020/v1

License: (c) (i) This work is licensed under a Creative Commons Attribution 4.0 International License. Read Full License 
1 Title: Peri-Operative Cerebral Regional Oxygen Saturation Variability and Short-Term

2 Outcomes Following Cardiac Surgery in Neonates

3 Maryam O. Abubakar ${ }^{1}$, Santina A. Zanelli ${ }^{1}$, Michael C. Spaeder ${ }^{2}$

4

5 Affiliations:

$6{ }^{1}$ Division of Neonatology, Department of Pediatrics, University of Virginia, Charlottesville,

7 VA,22908, USA

$8 \quad{ }^{2}$ Division of Pediatric Critical Care, Department of Pediatrics, University of Virginia,

9 Charlottesville, VA,22908, USA

10 Corresponding Author:

11 Maryam O Abubakar, MD

12 ORCID: 0000-0001-9332-0852

13 University of Virginia Hospital, PO Box 800386

14 Charlottesville VA 22908.

15 Email: moa3yr@ virginia.edu

16

\section{Impact Statement:}

- Cerebral regional oxygen saturation $\left(\mathrm{crSO}_{2}\right)$ is a marker of cerebral perfusion

19 autoregulation 
Decreased post-operative cerebral region oxygenation saturation $\left(\mathrm{crSO}_{2}\right)$ variability, a

41 surrogate for cerebral autoregulation, correlates with poor neurodevelopmental outcomes in

42 neonates who undergo cardiac surgery. The goal of this study is to investigate the relationship

43 between pre- and post-operative $\mathrm{crSO}_{2}$ variability in neonates requiring neonatal cardiac surgery

44 for congenital heart disease (CHD).

The variability of averaged 1-min $\mathrm{crSO}_{2}$ values was calculated for a minimum of $12 \mathrm{~h}$

46 before and for the first $48 \mathrm{~h}$ following cardiac surgery with cardiopulmonary by-pass in neonates

47 between November 2019 and May 2021.

The $\mathrm{CrSO}_{2}$ variability increased by $9 \%$ with each additional postnatal day in the pre-

49 operative monitoring period $(\mathrm{p}=0.009)$. There was a $40 \%$ decrease in $\mathrm{crSO}_{2}$ variability between

50 the pre-and post-operative monitoring periods $(\mathrm{p}<0.001)$. There were no associations between

51 the degree of decrease in $\mathrm{crSO}_{2}$ variability and CHD classification (aortic arch obstruction or

52 single ventricle physiology).

The $\mathrm{crSO}_{2}$ variability improves with each additional postnatal day but then decreases by

54 almost half following cardiac surgery in neonates. We did not observe any association between

55 pre-operative $\mathrm{crSO}_{2}$ variability and post-operative ventilator-free days, post-operative ICU

56 days, or mortality. The long-term effects or significance of reduced $\mathrm{crSO}_{2}$ require further

57 exploration. 


\section{INTRODUCTION}

63 Near infrared spectroscopy (NIRS) has been in use for more than four decades in the field of

64 critical care and is increasingly used in the neonatal intensive care unit (NICU). ${ }^{1}$ NIRS allows for

65 the non-invasive monitoring of tissue oxygenation based on the physiologic property of light

66 adsorption of hemoglobin at different wavelengths and provides a surrogate for blood flow and

67 its fluctuations. A major clinical application of NIRS is the peri-operative monitoring of neonates

68 with congenital heart disease (CHD). ${ }^{2,3}$

69 While the childhood mortality of patients with CHD has significantly decreased,

70 neurodevelopmental impairments remain one of the most common complications affecting

71 survivors of CHD. ${ }^{4,5}$ As such, the identification of patient-specific risk factors and/or modifiable

72 management strategies to improve outcomes in neonates undergoing neonatal cardiac surgery is

73 a high research priority. ${ }^{6,7}$

74 Few studies have explored the absolute trends in cerebral regional oxygen saturation $\left(\mathrm{crSO}_{2}\right)$ in

75 infants prior to surgery for CHD. These studies indicate that pre-operative $\mathrm{crSO}_{2}$ is lower in

76 infants with CHD compared to healthy controls, especially in those with left-sided obstructive

77 lesions. ${ }^{8-10}$ Evidence also supports an association between peri-operative $\mathrm{crSO}_{2}$ and

78 neurodevelopmental outcomes in infants with CHD. ${ }^{11}$ However, there remains significant gaps in

79 knowledge regarding postnatal baseline $\mathrm{crSO}_{2}$ normal range as well as its impact on

80 neurodevelopmental outcomes in neonates with CHD. 
81 Beyond absolute $\mathrm{crSO}_{2}$ trend, $\mathrm{crSO}_{2}$ variability, which indirectly reflect cerebral autoregulation,

82 sheds light into cerebral perfusion. ${ }^{12}$ Previous studies have found correlations between cerebral

83 NIRS wave variability and blood pressure variability in preterm infants as well as a strong

84 correlation between slow cerebral NIRS waveform and slow transcranial doppler in neonates

85 with sepsis. ${ }^{13.14}$ Changes in $\mathrm{crSO}_{2}$ variability, specifically a decrease in post-operative $\mathrm{crSO}_{2}$

86 variability amongst survivors of CHD surgery, have been linked with poor neurodevelopmental

87 outcomes particularly in neonates with aortic arch obstruction repair. ${ }^{3,15}$

88 The goal of this study is to investigate the relationship between pre- and post-operative $\mathrm{crSO}_{2}$

89 variability in a cohort of neonates undergoing cardiac surgery for CHD. The quantification of

$90 \mathrm{crSO}_{2}$ variability during the peri-operative period is important to better understand the impact of

91 pre- and post-operative factors (e.g., perfusion technique) on $\mathrm{crSO}_{2}$ variability and outcomes in

92 this high-risk population.

\section{METHODS}

94 This study was approved and exempted from a full review by the Institutional Review Board

95 (IRB) at the University Of Virginia School Of Medicine. A patient consent requirement was also

96 waived by the Institutional Review Board (IRB) at the University of Virginia School of

97 Medicine. Every aspect of this research was performed in accordance with relevant

98 guidelines/regulations. We included neonates ( $<30$ days of age on admission and $\geq 35$ weeks'

99 gestation at time of delivery) that underwent cardiac surgery with cardiopulmonary by-pass

100 (CPB) in the first 30 days after birth from November 2019 to May 2021. All patients underwent

101 bi-caval venous cannulation and those patients requiring neonatal arch repair underwent

102 anterograde cerebral perfusion (ACP), with either brief or no exposure to deep hypothermic

103 circulatory arrest (DHCA). 
104 Pre-operative $\mathrm{crSO}_{2}$ monitoring took placed either in the NICU or in the pediatric intensive care unit (PICU), no earlier than the second day after birth and for a minimum period of $12 \mathrm{~h}$. Postoperative $\mathrm{crSO}_{2}$ monitoring occurred in the PICU for the first $48 \mathrm{~h}$ following surgery. The $\mathrm{crSO}_{2}$ values were continuously captured using either the Foresight Elite ${ }^{\circledR}$ tissue oximeter (Edwards Lifesciences, Irvine, CA) for patients who had their pre-operative monitoring in the NICU, or the INVOS ${ }^{\mathrm{TM}} 5100 \mathrm{C} \AA$ cerebral oximeter (Medtronic, Minneapolis, MN) for all infants cared for in the PICU. crSO2 values were averaged over 1-min intervals and $\mathrm{crSO}_{2}$ variability was calculated using the root mean of successive squared differences (RMSSD) for both the pre- and postoperative phases of monitoring as previously described. ${ }^{3}$ In practical terms, the RMSSD measures the amount of change in $\mathrm{crSO}_{2}$ from minute-to-minute over the course of a specified period. Missing $\mathrm{crSO}_{2}$ values were imputed using predictive mean matching. To characterize changes in $\mathrm{crSO}_{2}$ variability over time, we also calculated a 60 -min moving average measure for each minute of monitoring using the RMSSD from the previous $60 \mathrm{~min}$.

Patient and clinical characteristics were collected, including age at time of admission to the NICU, age at time of pre-operative $\mathrm{crSO}_{2}$ monitoring start, age at time of surgery, weight at admission and time of surgery. In addition, patients were assigned to one of four previously described diagnostic classes of CHD: class 1-two ventricle physiology without aortic obstruction, class 2 - two ventricle physiology with aortic obstruction, class 3 - single ventricle physiology without aortic obstruction, or class 4-single ventricle physiology with aortic obstruction. ${ }^{16}$

The primary clinical outcomes of interest were ventilator-free days, defined as the number of days without invasive mechanical ventilation following surgery to post-operative day 28 , with 
126 patients who died before day 28 while on mechanical ventilation assigned zero, mortality, post-

127 operative seizures, and post-operative ICU days.

128 Distribution of continuous variables were assessed using the Wilk-Shapiro test for normality.

129 Continuous variables were compared using Student's $t$ test, Wilcoxon signed rank test, or linear

130 regression as appropriate. Pre- and post-operative $\mathrm{crSO}_{2}$ measures were matched at the patient

131 level and compared using paired difference $t$ test or Wilcoxon matched-pairs signed rank test as

132 appropriate. Categorical variables were compared using chi-square test or Fisher exact test as

133 appropriate. Type I error was set at 0.05 . All calculations were performed using STATA/IC 12.1

134 (STATA Corporation, College Station, TX).

\section{RESULTS}

\section{Cohort characteristics}

137 Thirty-seven neonates were included in the study with a median of 7 days of age (interquartile 138 range (IQR) 6-10 days) at the time of surgery. The cohort's demographic and clinical 139 characteristics are described in Table 1.

140 Primary Outcomes

141 We did not observe any association between pre-operative $\mathrm{crSO}_{2}$ variability and post-operative 142 ventilator-free days, post-operative ICU days, or mortality (Table 1).

143 Pre-operative $\mathrm{crSO}_{2}$

144 The median age at the start of pre-operative $\mathrm{crSO}_{2}$ monitoring was 4 days (IQR 2 - 5 days). We 145 observed an increase in the $\mathrm{crSO}_{2}$ variability of $9 \%$ with each additional day after birth in the 146 pre-operative monitoring period $(\mathrm{p}=0.009)$. There were no differences in pre-operative $\mathrm{crSO}_{2}$ 
147 variability based on CHD classification: aortic arch obstruction (classes 2 and 4) or single

148 ventricle physiology (classes 3 and 4). There were no differences in types of cardiac lesion or

149 clinical characteristics between patient monitored pre-operatively in the NICU versus PICU (or

150 based on monitor used).

151 The $\mathrm{crSO}_{2}$ variability was $31 \%$ lower in patients receiving invasive mechanical ventilation

152 during the pre-operative monitoring phase $(\mathrm{p}=0.04) . \mathrm{crSO}_{2}$ variability was not affected by the

153 need for continuous infusions for pain and/or sedation $(\mathrm{p}=0.25)$.

154 Post-operative $\mathrm{CrSO}_{2}$

155 Variability of $\mathrm{crSO}_{2}$ decreased by $40 \%$ between the pre- and post-operative monitoring period 156 ( $\mathrm{p}<0.001)$. There were no associations between the degree of decrease in $\mathrm{crSO}_{2}$ variability and 157 CHD classification (aortic arch obstruction vs. single ventricle physiology). There were no 158 differences in the mean absolute $\mathrm{crSO}_{2}$ values between the pre- and post-operative periods $(72 \%$ $159 \mathrm{vs} 74 \%$ ). The time series plots of pre- and post-operative $\mathrm{crSO}_{2}$ values and corresponding $\mathrm{crSO}_{2}$ 160 variability for a representative neonate are shown in Figure 1.

162 Table 1. Patient demographics and clinical characteristics.

\begin{tabular}{|l|l|}
\hline Characteristics & $\mathrm{n}(\%)$ \\
\hline Age at time of surgery (days) & 7 (IQR 6 - 10) \\
\hline Gestational age (weeks) & $39(\mathrm{IQR} 38-39)$ \\
\hline Birth weight (kg) & $3.3(\mathrm{IQR} 2.9-3.6)$ \\
\hline
\end{tabular}




\begin{tabular}{|l|l|}
\hline Female sex & $17(46 \%)$ \\
\hline Class 1 (two ventricles, no aortic arch obstruction) & $11(30 \%)$ \\
\hline Class 2 (two ventricles, aortic arch obstruction) & $13(35 \%)$ \\
\hline Class 3 (single ventricle, no aortic arch obstruction) & $2(5 \%)$ \\
\hline Class 4 (single ventricle, aortic arch obstruction) & $11(30 \%)$ \\
\hline Age at start pre-operative monitoring (days) & $4(\mathrm{IQR} 2-5)$ \\
\hline Mechanical ventilation during pre-operative & $9(24 \%)$ \\
\hline monitoring & \\
\hline Continuous sedation during pre-operative monitoring & $5(14 \%)$ \\
\hline CPB time (min) & $166(\mathrm{IQR} 141-188)$ \\
\hline Cross-clamp time (min) & $92(\mathrm{IQR} 66-131)$ \\
\hline Post-operative seizures & $1(3 \%)$ \\
\hline Ventilator-free days & $23(\mathrm{IQR} 19-25)$ \\
\hline Post-operative ICU days & $3(8 \%)$ \\
\hline Mortality & \\
\hline
\end{tabular}


167 In this cohort of neonates with CHD requiring neonatal cardiac surgery and CBP, there was no 168 difference in the mean absolute $\mathrm{crSO}_{2}$ between the pre- and post-operative periods. However, 169 there was a significant decrease in $\mathrm{crSO}_{2}$ variability between the pre- and immediate post170 operative period suggesting alterations in cerebral autoregulation which do not impact the 171 absolute $\mathrm{crSO}_{2}$ trends. Whether or not the duration and degree of post-operative $\mathrm{crSO}_{2}$ variability

172 impacts long-term neurodevelopmental outcomes remains unknown.

173 CBP allows surgeons to carry out intracardiac repairs of CHD by providing an alternate means of

174 circulation and gaseous exchange without blood flowing into the heart or lungs. During CPB, 175 deoxygenated blood returning to the heart is channeled to an extracorporeal oxygenator for gas 176 exchange and subsequently returned via a cannula into the ascending aorta. ${ }^{17}$ However, total 177 cessation of circulation is necessary in some surgical repairs especially those requiring aortic 178 arch reconstruction to allow for a bloodless surgical field. In such repairs, DHCA has 179 traditionally been used to cool patients to a temperature range of $15-20^{\circ} \mathrm{C}$ to reduce metabolic 180 demands. ${ }^{17}$ Newer techniques such as ACP allow maintenance of only cerebral perfusion during 181 such complex repairs often shortening or eliminating of the need for DHCA. ${ }^{17.18}$ Animal studies 182 indicate that CPB leads to alterations in tissue oxygenation and hemoglobin parameters. ${ }^{19,20}$ Our 183 data suggest that alterations in cerebral autoregulation occur in the peri-operative period in 184 neonates requiring surgery for CHD. The impact of these changes in unknown, but concerns 185 remain that these alteration in cerebral autoregulation may adversely impact neurodevelopment 186 in these patients. Future studies should include the investigation of additional intra-operative 187 variables including anesthesia type as well as use of DHCA vs ACP. 
188 An interesting finding in this study was the improvement in the pre-operative $\mathrm{crSO}_{2}$ variability

189 observed with each postnatal day prior to surgery in these neonates. Cerebral blood flow and

190 hemodynamics are known to improve over time as neonates transition from fetal to extra-uterine

191 circulation. Hence this a physiologically plausible finding. Considering the significant drop in

$192 \mathrm{crSO}_{2}$ variability after cardiac surgery, a robust variability prior to surgery may be desirable.

193 Whether improved pre-operative $\mathrm{crSO}_{2}$ variability is associated with improved

194 neurodevelopmental outcomes and could be used to optimize surgery timing is unknown and

195 should be further studied.

196 Our study has several limitations including its small and single center design. In addition, we

197 were not able to include other vital sign parameters in our analysis. Future multicenter

198 longitudinal studies exploring other important markers of perfusion such as mean arterial blood

199 pressure variability, heart rate variability in addition to $\mathrm{crSO}_{2}$ variability may further advance our

200 understanding of the relationship between cerebral autoregulation and outcomes in neonates with

201 CHD. Another limitation of this study is that two different NIRS monitors were utilized -the

202 Foresight Elite ${ }^{\circ}$ tissue oximeter (Edwards Lifesciences, Irvine, CA) pre-operatively and the

203 INVOS ${ }^{\text {TM }} 5100 \mathrm{C} \AA$ cerebral oximeter (Medtronic, Minneapolis, MN). Our measurement of

204 interest - $\mathrm{CrSO}_{2}$ variability is generally agnostic to the absolute value of the measured

205 parameter. $^{3}$

206 CONCLUSION

$207 \mathrm{crSO}_{2}$ variability, which is a marker of cerebral autoregulation, decreases significantly in

208 neonates with CHD after surgical repair involving CPB. Further research is needed to explore the 209 long-term effects of this post-surgical drop in $\mathrm{crSO}_{2}$ variability. 


\section{REFERENCES}

213 1. Garvey, A. A., \& Dempsey, E. M. Applications of near infrared spectroscopy in the

214 neonate. Current opinion in pediatrics, 30(2), 209-215.

215 doi:https://doi.org/10.1097/MOP.0000000000000599(2018).

216 2. McKenzie, E. D., Andropoulos, D. B., DiBardino, D., \& Fraser, C. D. Congenital Heart

217 Surgery 2005: The brain: It's the heart of the matter. The American Journal of Surgery, 190(2),

218 289-294. doi: https://doi.org/10.1016/J.AMJSURG.2005.05.029(2005).

219 3. Spaeder, M. C., Klugman, D., Skurow-Todd, K., Glass, P., Jonas, R. A., \& Donofrio, M.

220 T. Perioperative Near-Infrared Spectroscopy Monitoring in Neonates with Congenital Heart

221 Disease: Relationship of Cerebral Tissue Oxygenation Index Variability with

222 Neurodevelopmental Outcome. Pediatric Critical Care Medicine, 18(3), 213-218.

223 https://doi.org/10.1097/PCC.0000000000001056 (2017).

224 4. Gaynor, J. W., Stopp, C., Wypij, D., Andropoulos, D. B., \& Atallah, J.

225 Neurodevelopmental Outcomes After Cardiac Surgery in Infancy. Pediatrics, 135(5), X3-X3.

226 https://doi.org/10.1542/peds.2014-3825d (2015).

227 5. Cassidy A.R., White M.T., DeMaso D.R., Newburger J.W., Bellinger D.C. Executive 228 Function in Children and Adolescents with Critical Cyanotic Congenital Heart Disease. J Int 229 Neuropsychol Soc. 2015; 21(1):34-49. doi:10.1017/S1355617714001027 
230 6. Gaynor, J. W., et al. Patient characteristics are important determinants of

231 neurodevelopmental outcome at one year of age after neonatal and infant cardiac surgery. The

232 Journal of thoracic and cardiovascular surgery, 133(5), 1344-1353.e13533.

233 https://doi.org/10.1016/j.jtcvs.2006.10.087. (2007).

234 7. Bellinger, D. C., et al. Developmental and neurologic status of children after heart 235 surgery with hypothermic circulatory arrest or low-flow cardiopulmonary bypass. The New 236 England journal of medicine, 332(9), 549-555. https://doi.org/10.1056/NEJM199503023320901

237 8. Dehaes, M.,et al. Perioperative cerebral hemodynamics and oxygen metabolism in 238 neonates with single-ventricle physiology. Biomedical optics express, 6(12), 4749-4767. 239 https://doi.org/10.1364/BOE.6.004749. (2015).

2409 Uebing, A., et al. Perioperative cerebral and somatic oxygenation in neonates with 241 hypoplastic left heart syndrome or transposition of the great arteries. The Journal of thoracic and 242 cardiovascular surgery, 142(3), 523-530. https://doi.org/10.1016/j.jtcvs.2011.01.036 (2011).

243 10. Mebius, M. J., Kooi, E. M. W., Bilardo, C. M., \& Bos, A. F. Brain Injury and 244 Neurodevelopmental Outcome in Congenital Heart Disease: A Systematic Review. Pediatrics, 245 140(1), e20164055. https://doi.org/10.1542/peds.2016-4055(2017).

246 11. Toet, M. C. et al. Cerebral oxygen saturation and electrical brain activity before, during, 247 and up to 36 hours after arterial switch procedure in neonates without pre-existing brain damage: 248 its relationship to neurodevelopmental outcome. Experimental brain research, 165(3), 343-350. 249 https://doi.org/10.1007/s00221-005-2300-3(2005). 
250 12. Brady K. et al: Real-time continuous monitoring of cerebral blood flow autoregulation

251 using near-infrared spectroscopy in patients undergoing cardiopulmonary bypass. Stroke 2010;

$252 \quad 41: 1951-1956$

253 13. Tsuji M. et al. Cerebral intravascular oxygenation correlates with mean arterial pressure 254 in critically ill premature infants. Pediatrics. 2000; 106:625-632. [PubMed: 11015501]

255 14. Steiner L., Pfister D., Strebel S., Radolovich D., Smielewski P., Czonsnyka M. Near256 infared spectroscopy can monitor dynamic cerebral autoregulation in adults. Neurocrit Care. 2572008 Sep. Epub.

258 15. Spaeder, M.C., \& Surma, V.J. Cerebral regional oxygen saturation variability in neonates 259 following cardiac surgery. Pediatr Res .https://doi.org/10.1038/s41390-020-01171-1. (2020).

260 16. Clancy R R. et al: Preoperative risk of death prediction model in heart surgery with deep 261 hypothermic circulatory arrest in the neonate. J Thorac Cardiovasc Surg 119,347-357(2000)

262 17. Ginther, R. M. \& Forbess, J. M. in Fuhrman \& Zimmerman's Pediatric Critical Care

263 (eds Fuhrman, B. P. \& Zimmerman, J. J.) 430-446 (Elsevier, Philadelphia, 2017).

264 18. Fraser, C. D. \& Andropoulos, D. B. Principles of antegrade cerebral perfusion

265 during arch reconstruction in newborns/infants. Semin. Thorac. Cardiovasc. Surg.

266 Pediatr. Card. Surg. 11, 61-68 (2008).

267 19. Abdul-Khaliq, H. et al. Dynamic changes in cerebral oxygenation related to deep

268 hypothermia and circulatory arrest evaluated by near-infrared spectroscopy. Acta

269 Anaesthesiologica Scandinavica, 45: 696-701. https://doi.org/10.1034/j.1399-

$270 \quad \underline{6576.2001 .045006696 . x}(2001)$ 
271 20. Votava-Smith J.K. et al. Impaired cerebral autoregulation in preoperative newborn

272 infants with congenital heart disease. J. Thorac. Cardiovasc. Surg 154, 1038-1044 (2017).

273 21. Gagnon R.E. et al: Comparison of two spatially resolved NIRS oxygenation indices. J

274 Clin Monit Comput17,385-291(2002)

275 


\section{AUTHOR CONTRIBUTIONS}

279 M.O.A. made substantial contributions to the design, data acquisition and drafting of article.

280 M.C.S. made substantial contributions to the concept, design, data acquisition, analysis and

281 interpretation of data and drafting of article.

282 S.A.Z. made substantial contributions to the data acquisition and review of draft and data

283 interpretation.

284 ADDITIONAL INFORMATION

285 Financial Support: University of Virginia, Department of Pediatrics, Division of Neonatology

286 Conflicts/Disclosures: None

287 Category of Study: Clinical Research

288 Patient Consent: Waived by the Institutional Review Board of the University Of Virginia 289 School Of Medicine. 
Figures
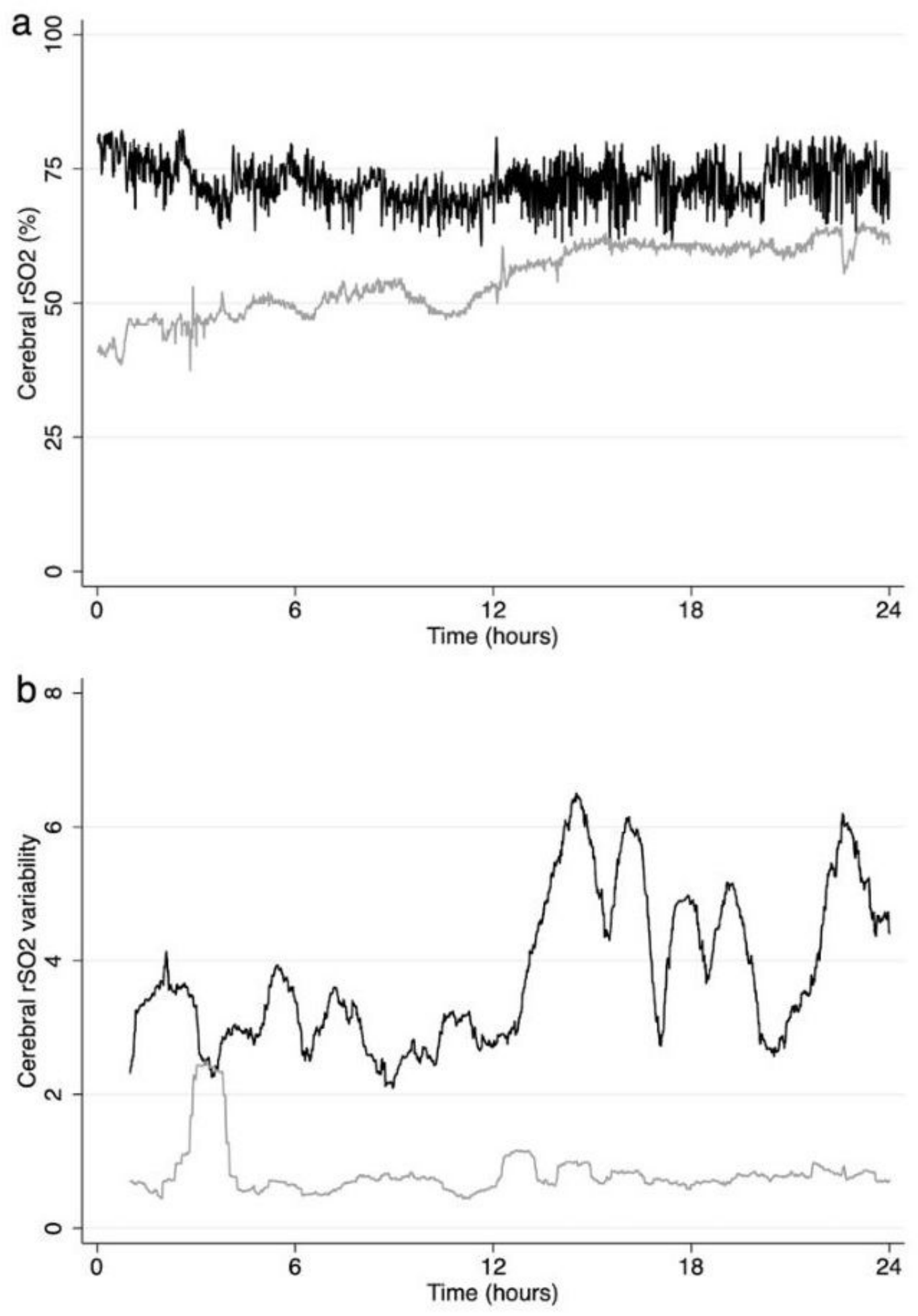

Figure 1

Time series plot of pre- and post-operative crSO2 values and corresponding crSO2 variability for a representative neonate.Time series plot of a crSO2 values and b crSO2 variability for patient with hypoplastic left heart syndrome captured pre- (black) and post- (grey) Norwood/Sano palliation. 